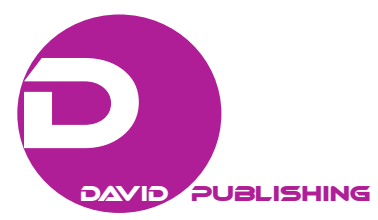

\title{
Smart Rehabilitation Solutions Through IoT and Mobile Devices
}

\author{
Raúl Morales Salcedo, Milton Carlos Elías Espinosa \\ Tecnológico de Monterrey, Nuevo León, Mexico
}

\begin{abstract}
Hand injuries are a frequent problem in actual society. These are often caused while working or exercising, but simple everyday activities can also be the cause. The most common injuries are those related to soft tissue; specifically, muscles, tendons, and ligaments. To improve therapy results and reduce its cost and needed time, a self-rehabilitation system for soft tissue injuries in the hands was developed. The proposed system is meant to be implemented on Smart TVs and can be used in the home of the injured people. To identify the most common movements of the injured limbs, and design proper therapies to be displayed by the system, an exoskeleton prototype which supports four degrees of freedom of the hand was constructed. We examined scenarios to provide services for individuals and created a scenario-based prototype, which was tested by an exercise therapist to verify compliance with the requirements of users.
\end{abstract}

Keywords: rehabilitation-training system, mechatronics, Smart TV, system mobile rehabilitation

\section{Introduction}

Self-rehabilitation is not a new concept; work in this area can be found for its use in cardiovascular and pulmonary disease (Hesse, Schmidt, Werner, \& Bardeleben, 2003; Curkendall, deLuise, \& Jones, 2006), and stroke (Feng, Liu, \& Guo, 2013). Computer visualization applications are now popular for game-based rehabilitation (Norouzi-Gheidari, Levin, Fung, \& Archambault, 2013) but operating a computer can be troublesome for those people with body injuries. Thus, a simpler and more familiar self-device is preferred. Smart TVs are a familiar medium which has previously been used for home care. In 2010, a prototype was developed for the provision of home care services based on interactive television technology called iTV (López de Ipiña, Blanco, Laiseca, \& Díaz de Sarrelde, 2010). The goal of iTV was to facilitate communication and monitoring with various appliances using a graphical and intuitive interface. In the same way, smart agents have been used to regulate flow of data, and users can check their own health records through a TV screen or even have a digital meeting with his doctor (Donhee, Jae-Gil, \& Hong-Seok, 2014; Martinez-Pabon, Caicedo-Guerrero, Ibarra-Samboni, Ramirez-Gonzalez, \& Hernández-Leo, 2015).

Smart television services for the provision of health care in the family environment have become popular and have the potential to provide health and social care to people in their own homes (Prey et al., 2014). Several smart devices have been used for vital health monitoring (Fernandes, Afonso, \& Simões, 2011; Biswas \& Mira, 2015). This technology allows elderly users to inspect numerical or graphical summaries of the

Raúl Morales Salcedo, Ph.D., professor, Department of Computing, Tecnológico de Monterrey, Santa Fe, México.

Milton Carlos Elías Espinosa, Ph.D., professor, Department of Mechanics and Advanced Materials, Tecnológico de Monterrey, Santa Fe, México.

Correspondence concerning this article should be addressed to Raúl Morales Salcedo, Department of Computing, School of Engineering and Sciences, Tecnológico de Monterrey, Av. de los Poetas 100, Santa Fe, La Loma, 01389, México. 
data for specific time periods or for unusual events using a smart TV set. The data are displayed on a Smart TV set connected to a network, which allows inspection of more complex data but requires a larger screen and more convenient methods of interaction. We propose a rehabilitation training system attached to a Smart TV and IoT sensors to create a good home environment for those who needs treatment of rehabilitation for hand injuries.

\section{Rehabilitation Training System}

To produce a functional rehabilitation training system, there are numerous design and manufacturing challenges to overcome. The challenge in this work was to create a modular, lightweight, reasonably complex, and quality device which was easily attached even to deformed or scarred hands, and that could be further used for research in the field of mobile rehabilitation training systems.

\section{Construction}

Design. The design of an exoskeleton for the mobile rehabilitations training system (see Figure 1) mimics the human arm and the amount of dexterity it can offer. It supports bidirectional motion in all joints.

Control. A device is easy and natural to control by the user. The prototype's controller consists of three parts: a Raspberry Pi running routines with sequences of rehabilitation movements, Raspbian as operating system allowing hardware and software communication, and the client interface giving visual feedback to the patient. Patient interface can be used on a Smart TV or a computer if desired.

Viability. The device must be useful to any person with hand injuries. Whether or not this device is ever used by people with hand injuries, the goal is to develop a mobile device with Smart TV connectivity which can benefit them.

Reasonably priced. We aim to keep the material cost of the device as low as possible. Modern commercial exoskeleton for arms generally cost from $\$ 9,000$ to $\$ 12,000$. Material should cost for this design will be under $\$ 400$.

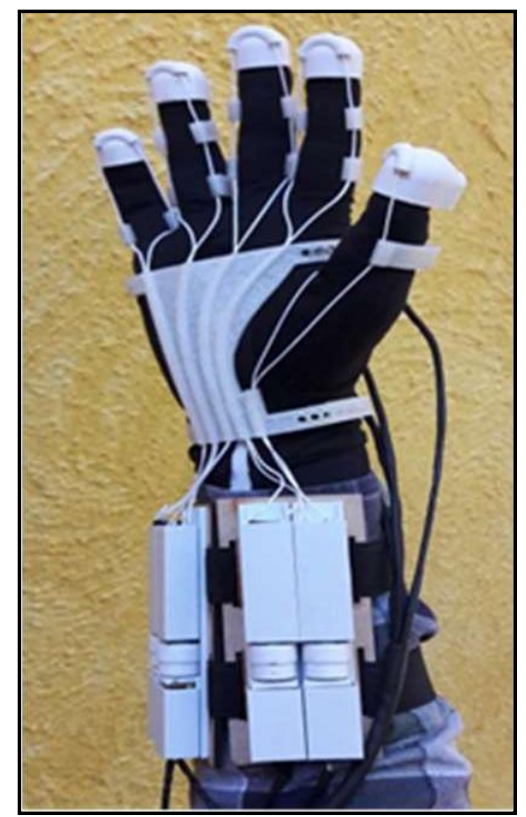

Figure 1. Prototype of the exoskeleton for the hand. 


\section{Experimental Setup and Data Acquisition}

For the scope of the present study, we monitored three of system mobile rehabilitation's wearable modules: index finger (IF), thumb (TH), and hand dorsum (DM) (see Figure 2). The mobile rehabilitation system transmitted a powered motion from external actuators to its modules without constraints between anatomical and robotic joint axes.

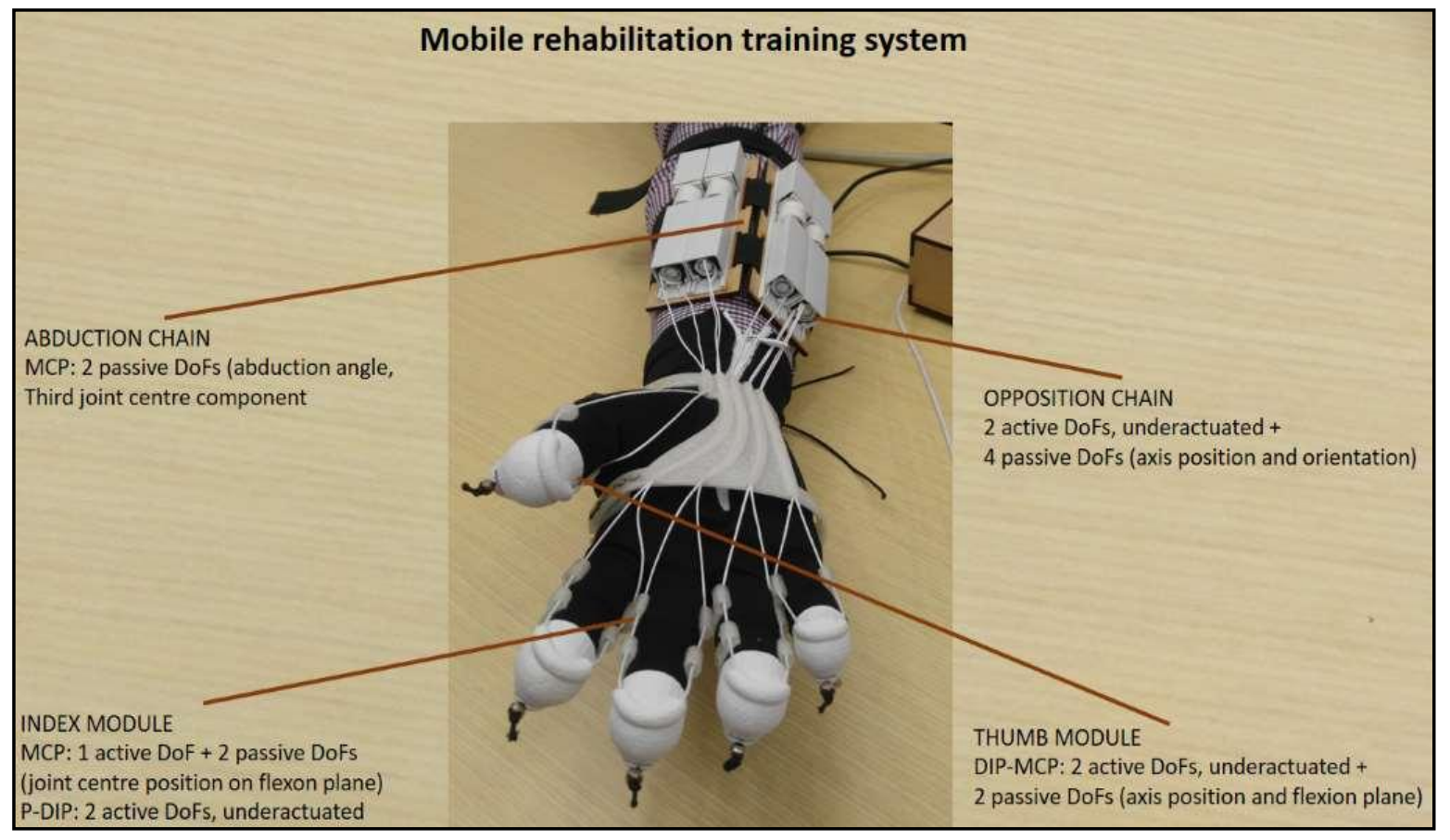

Figure 2. Overview of the mobile rehabilitation system.

A motion capture (MOCAP) system was used to capture the 3D trajectories of hemispheric passive markers moving in a calibrated volume of $600 \times 400 \times 300 \mathrm{~mm}, \mathrm{X}, \mathrm{Y}$, and Z. This system was used to measure the user's kinematics since the hand segments were mostly covered by the HX itself. Specifically, the following positioning for the markers was used (see Figure 3):

Three different grips (palmar grasp, pinch, and key grip) were tested in different operating conditions: (1) active (normal), the mobile rehabilitation system moved the hand of the participant; (2) passive (normal), the participant moved their hand while wearing the unlatched mobile rehabilitation system; (3) passive (no hand movement), global free motion of the upper arm, without moving fingers, hand, or wrist; and (4) passive (hand-full-opening), the participant fully opened their hand, reaching a complete extension of the fingers. Five trials were performed for each condition. Six healthy adult volunteers participated on the experiments with their age, weight, and height being $39.5 \pm 9.1$ years, $69.7 \pm 7.5 \mathrm{~kg}$, and $1.73 \pm 0.05 \mathrm{~m}$ (mean $\pm \mathrm{SD}$ ) respectively. All the participants gave written informed consent approved by the Instituto Nacional de Rehabilitación Luis Guillermo Ibarra Ibarra, México. 


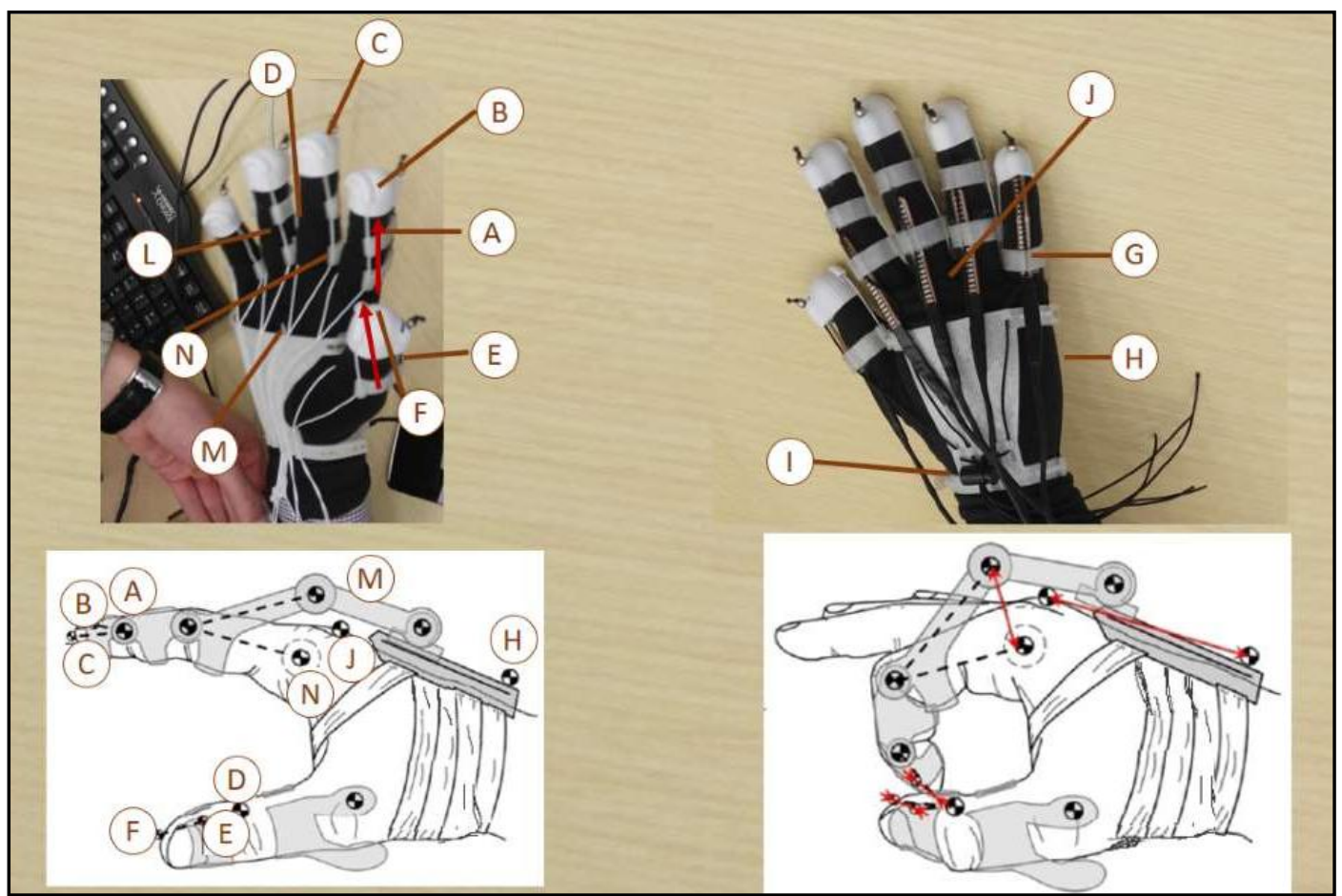

Figure 3. Markers and relative displacement measures. Positions of markers on the thumb and index finger (left) and on the hand dorsum (right). The figure represents the distances whose SD variability constitutes the analyzed numerical indexes.

\section{Data Visualization}

The 3D visual representation of the patients' movements is generated using Samsung SDK, Node.js, Python, and Pygame associated with the motion capture system (sensors IoT ready). The relative displacements of the Mobile rehabilitation system's modules were computed as follows:

(a) DM: standard deviation of the relative displacement between markers $\mathrm{E}$ and $\mathrm{F}$;

(b) DI-d: standard deviation of the relative displacement between markers B and C;

(c) DI-p: standard deviation of the normal distance of marker M from the index proximal phalanx;

(d) DM: the quadratic mean of the standard deviations of the $3 \mathrm{D}$ coordinates of marker $\mathrm{J}$ in the dorsum local reference frame.

Ideal values (a totally compliant mobile rehabilitation system) of these indexes were expected to be null. The relative displacement among markers placed on the same rigid frame, averaged over all conditions, was taken as the noise level of the experimental setup. A repeated measure ANOVA $(p<0.05)$ was performed using Fisher's LSD (least significant difference) post-hoc comparisons to evaluate the differences between conditions and between TH, IF-d, IF-p, and HD. Figure 4 shows the overall displacement indices for the different tests, and the noise average level $($ mean $=0.23 \mathrm{~mm})$ and upper limit $(\mathrm{CI}-95 \%=0.59 \mathrm{~mm})$. Figure 5 shows the analysis with ANOVA results indicated by horizontal lines joining statistically different data-sets. 


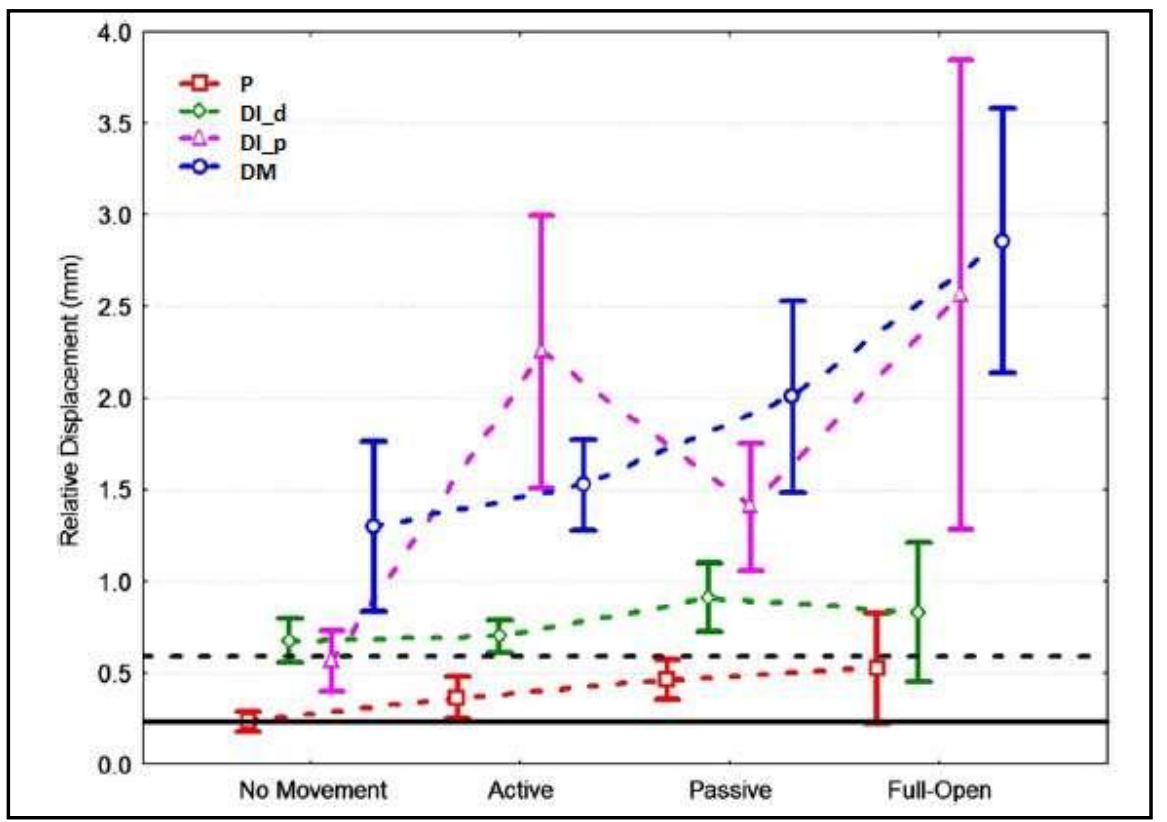

Figure 4. Relative displacement indexes for different modules and condition. Vertical bars represent the 0.95 confidence interval (corresponding to CI-95\%) of the mobile rehabilitation system modules displacement. Horizontal black lines represent noise level (solid is mean value, dotted is the CI-95\% upper limit value).

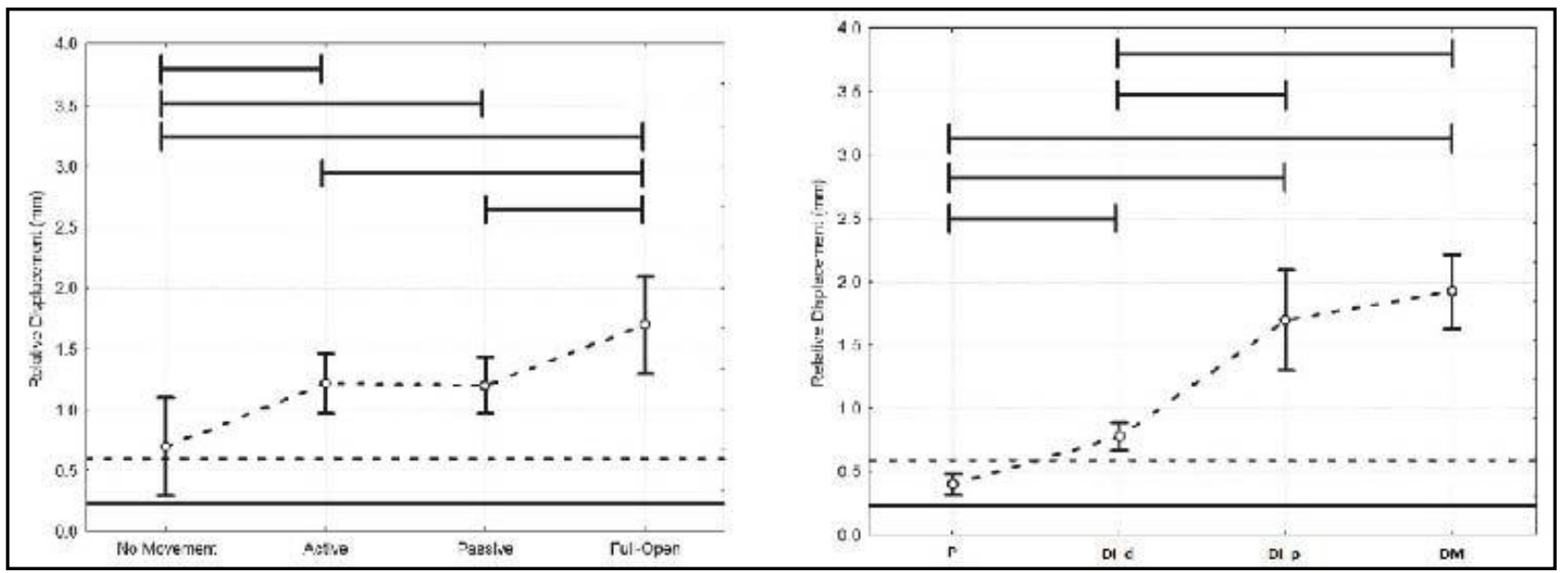

Figure 5. Statistical analysis. Results of statistical analysis (ANOVA, $p<0.05$; Fisher LSD post-hoc): effect of condition (top) and of the mobile rehabilitation system module (bottom). Statistically significant differences among conditions and modules are marked with horizontal lines.

\section{Results}

Statistical analysis showed that both conditions and hand segments had a significant effect on the mobile rehabilitation system wearing stability (see Figure 6). The DM and DI-p showed the greatest displacement, while the DI-d and the P were respectively just above and always within the noise band. As for the conditions, no-hand-movement and hand-full-opening showed the smallest and largest values respectively with the two functional tests in between.

DI-p was the only module showing larger "active" mode displacements than "passive" ones. DM relative displacements took into account the variability of the length of the vector joining the $\mathrm{J}$ and $\mathrm{H}$ markers; while 
the other measures were projections of marked vectors along given directions, the DM one included the three spatial components X, Y, and Z (see Figure 6). An additional analysis was performed on the DM results for the hand-full-opening test (the test with higher displacement). Figure 6 shows the relationships between the components of the relative movements and the grip aperture amplitude, the latter of which defined as the distance between the fingertip markers ( $\mathrm{C}$ and $\mathrm{F}$ ) for one of the six participants (representative of the others).
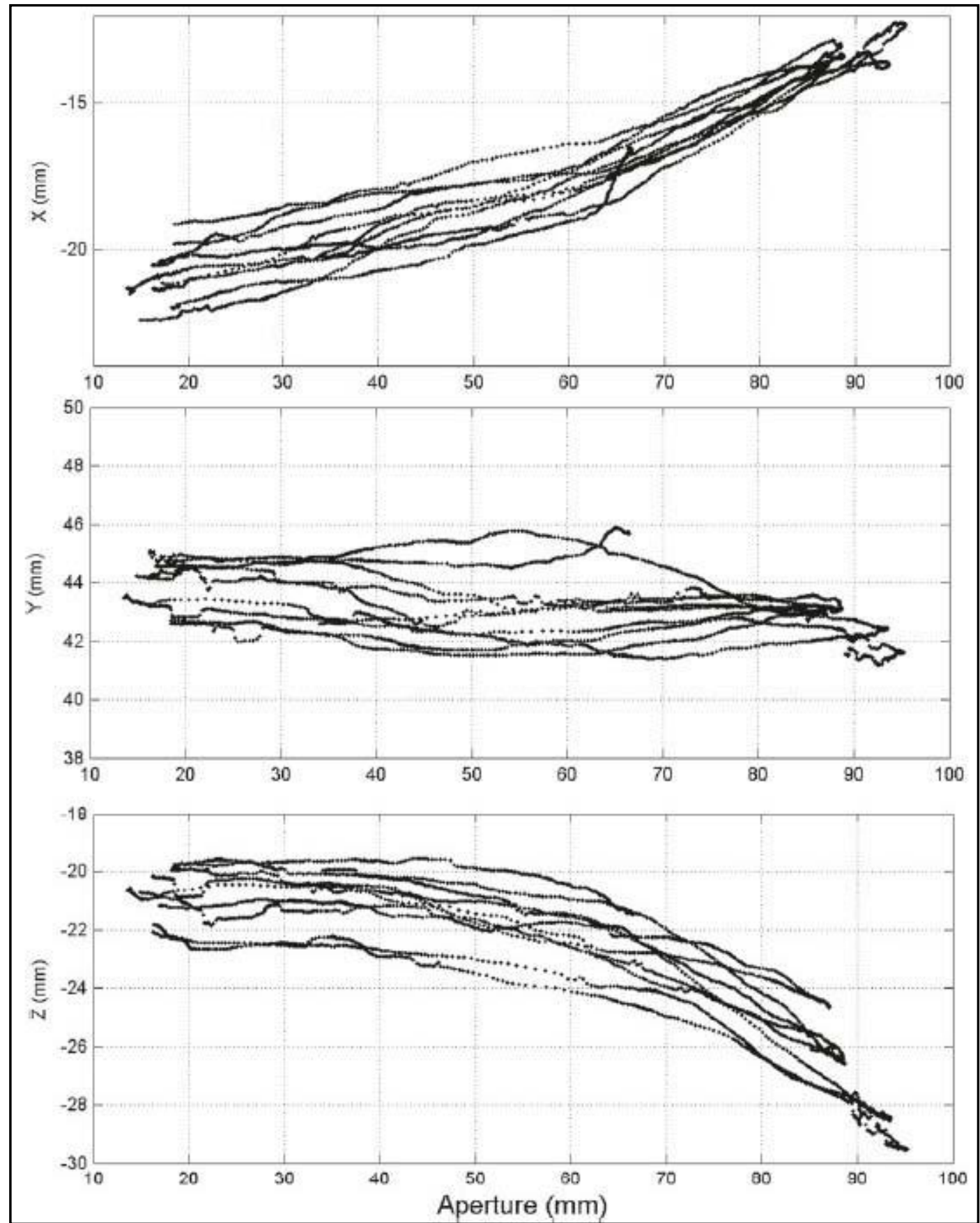

Figure 6. DM-user relative displacement as function of hand aperture. Example of X, Y, and Z components (in mm) of the relative displacement between the DM module and hand dorsum, as a function of grip aperture amplitude during the hand-full-opening task. 


\section{Conclusions}

Relative movements between the mobile rehabilitation system and the body segments are an indicator of user discomfort and lower assistance device effectiveness. The results of the present study showed that the stability of mobile rehabilitation system on the hand is optimal for the distal extremities of the actuated modules, even during the prospective therapeutic use of the device, where the assistance device drives the hand in executing grasps ("active" mode). We have shown the statistically significant reliability of the mobile rehabilitation system, through a quantitative analysis of hand and the assistance device relative movements, especially concerning the finger modules. Based on the presented results, future development of the mobile rehabilitation system will focus on refining the fixtures of the DM module, to stabilize its adherence. In the typical "active" operative mode, the displacements at the distal extremities were like those registered without motions, and close to the averaged noise level, suggesting that the device can be effective in functional grasp actuation for weak hands. Future studies will also deal with the improvement of the sheath-cable transmission system, limiting friction losses in joint design and feedback to the patient through a smart TV user interface.

\section{References}

Biswas, S., \& Mira, S. (2015). Designing of a prototype of e-health monitoring system. IEEE International Conference on Research in Computational Intelligence and Communication Networks (ICRCICN). November 20-22, Kolkata, India.

Curkendall, S., deLuise, C., \& Jones, K. (2006). Cardiovascular disease in patients with chronic obstructive pulmonary disease, Saskatchewan Canada cardiovascular disease in COPD patients. Annals of Epidemiology, 16(1), 63-70.

Donhee, D., Jae-Gil, L., \& Hong-Seok, Y. (2014). How does interactivity contribute to a smart TV user experience? In Proceedings of the 8th International Conference on Ubiquitous Information Management and Communication. January 9-11, New York, NY: ACM.

Feng, X., Liu, C., \& Guo, Q. (2013). Research progress in rehabilitation treatment of stroke patients: A bibliometric analysis. Neural Regeneration Research, 8(15), 1423-1430.

Fernandes, B., Afonso, J., \& Simões, R. (2011). Vital signs monitoring and management using mobile devices. IEEE 6th Iberian Conference on Information Systems and Technologies (CISTI). June 15-18, pp. 1-6.

Hesse, S., Schmidt, H., Werner, C., \& Bardeleben, A. (2003). Upper and lower extremity robotic devices for rehabilitation and for studying motor control. Current Opinion in Neurology, (16), 705-710. doi: 10.1097/00019052-200312000-00010

López de Ipiña, D., Blanco, S., Laiseca, X., \& Díaz de Sarrelde, I. (2010). Elder care: An interactive TV-based ambient assisted living platform. II International Workshop on Ambient Assisted Living (IWAAL-2010), September 7-10, Valencia, Spain.

Martinez-Pabon, F., Caicedo-Guerrero, J., Ibarra-Samboni, J., Ramirez-Gonzalez, G., \& Hernández-Leo, D. (2015). Smart TV-smartphone multiscreen interactive middleware for public displays. Scientific World Journal. doi: 10.1155/2015/534949. Retrieved from https://www.ncbi.nlm.nih.gov/pmc/articles/PMC4408749/

Norouzi-Gheidari, N, Levin M. F., Fung, J., \& Archambault, P. (2013). Interactive virtual reality game-based rehabilitation for stroke patients. International Conference on Virtual Rehabilitation (ICVR), pp. 26-29. doi: 10.1109/ICVR.2013.6662126

Prey, J. E., Woollen, J., Wilcox, L., Sackeim, A. D., Hripcsak, G., Bakken, S., .. Vawdrey, D. K. (2014). Patient engagement in the inpatient settings: A system review. Journal of the American Medical Informatics Associations, 20(4), 742-750. 